\title{
Psychological Distress in Quarantine Designated Facility During COVID-19 Pandemic in Saudi Arabia
}

This article was published in the following Dove Press journal: Risk Management and Healthcare Policy

\author{
Abdulmajeed A Alkhamees (D) \\ Moath S Aljohani ${ }^{2}$ \\ Mohammed A Alghesen ${ }^{3}$ \\ Ali T Alhabib ${ }^{4}$ \\ 'Department of Medicine, College of \\ Medicine and Medical Sciences, Qassim \\ University, Mlida, Al Qassim, Saudi \\ Arabia; ${ }^{2}$ Department of Family and \\ Community Medicine, College of \\ Medicine and Medical Sciences, Qassim \\ University, Mlida, Al Qassim, Saudi \\ Arabia; ${ }^{3}$ Psychiatry Mental Hospital, \\ Ministry of Health, Buraydah, Al Qassim, \\ Saudi Arabia; ${ }^{4}$ King Fahd Medical City \\ Academy for Postgraduate Studies in \\ Family Medicine, Ministry of Health, \\ Riyadh, Saudi Arabia
}

Background: Quarantine is a useful measure for preventing and controlling pandemics; however, it might be stressful for quarantined individuals. Fear and anxiety about a disease can be overwhelming. These emotions were reported for individuals in involuntary quarantine facilities dedicated to quarantine purposes.

Methods: This cross-sectional study surveyed the individuals in involuntary quarantine institutions (for a planned period of 14 days of quarantine) in two regions of Saudi Arabia. The mental health status of individuals was assessed using the Revised Impact of Event Scale (IES-R) and Depression, Anxiety, and Stress Scale (DASS-21).

Results: The study surveyed 214 quarantined/isolated individuals. The stress, anxiety, and depression rates were $25.7 \%, 21.5 \%$, and $32.7 \%$, respectively. On the IES-R, $28.0 \%$ of the participants met the criteria for psychological distress. Female gender, self-reported history of psychiatric disorder, and average health status were significantly associated with negative psychological impact and depression, anxiety, and stress symptoms $(\mathrm{p}<0.05)$. Watching television was found to be a factor in reducing rates on the IES-R and DASS-21 scale while working out lowered rates on the IES-R alone $(\mathrm{p}<0.05)$.

Conclusion: During an institutional involuntary quarantine, additional attention should be paid to vulnerable groups like females and individuals with a history of psychiatric illness. More than one-fourth of our sample experienced a negative psychological impact; therefore, coping practices like working out should be encouraged. This study contributes to the ongoing discussion about the psychological aspects of being quarantined. Much work remains to be done to identify strategies that prevent and mitigate psychological distress throughout the quarantine experience and to determine whether these impacts will last for an extended period of time.

Keywords: COVID-19, anxiety, IES, depression, knowledge, quarantine, psychological impact, stress, pandemic, Saudi Arabia

\section{Introduction}

The coronavirus disease 2019 (COVID-19) originated in China. It spread over the globe in just a few months, the number of infected people and deaths increasing as it went. Most countries in the world enacted partial or complete border closures to foreign nationals ${ }^{1}$ and recommended citizens to self-isolate at home or in a dedicated quarantine facility. ${ }^{2}$ Isolation and quarantine help protect the public by preventing exposure to people who have or may have a contagious disease. Isolation is the separation of sick people with a contagious disease from people
Correspondence: Abdulmajeed A Alkhamees

Department of Medicine, College of Medicine and Medical Sciences, Qassim University, Buraydah, Al Qassim, Saudi Arabia

Email a.alkhamees@qu.edu.sa
Risk Management and Healthcare Policy 2020:13 3103-3/20

smit your manuscript (c) (1) (-) 2020 Alkhamees et al. This work is published and licensed by Dove Medical Press Limited. The full terms of this license are available at https://www.dovepress.com/ C. the work you hereby accept the Terms. Non-commercial uses of the work are permitted without any further permission from Dove Medical $P$
For permission for commercial use of this work, please see paragraphs 4.2 and 5 of our Terms (https://www.dovepress.com/terms.php). 
who are not sick, and quarantine is the separation and restricted movement of people who have been exposed to a contagious disease while observing them for symptoms. Before the recent COVID-19 outbreak, cities were quarantined in areas of Canada and China during the severe acute respiratory syndrome (SARS) pandemic in 2003, and in South Korea in 2015 during the Middle East respiratory syndrome coronavirus (MERS-CoV). ${ }^{3}$ Quarantine has been confirmed as an effective tool for preventing and controlling an influenza pandemic. ${ }^{4}$ However, it may cause people high levels of stress. Fear and anxiety about a disease can be overwhelming. Previous studies also reported that quarantine could cause psychological harm; a study conducted in Taiwan investigated stress reactions among 338 staff members in a hospital and found that 5\% suffered from an acute stress disorder, $20 \%$ felt stigmatized and rejected in their neighborhood because of their hospital work, and 20 out of $218(9 \%)$ health-care workers reported reluctance to work or had considered resignation.${ }^{5}$ In another study conducted in Australia during an equine influenza outbreak, approximately $34 \%$ of participants reported high psychological distress, compared to levels of around $12 \%$ in the general Australian population. Another study in China during the SARS outbreak examined symptoms of depression in hospital staff three years after being quarantined and found $9 \%$ (48 of 549) of all sampled participants had high depressive symptoms. Of this group, $60 \%$ ( 29 of 48 ) of them had been quarantined, while only $15 \%$ (63 of 424) of participants with low depressive symptom had been quarantined.

During the current COVID-19 pandemic, a nationwide survey in Italy showed that female gender was associated with higher levels of psychological distress. Having an acquaintance infected with COVID-19 increased both depression and stress. A history of stressful situations and medical problems raised depression and anxiety levels as well. Some other factors found to increase anxiety and stress levels include having a family member infected with COVID-19, being young, and needing to leave one's home to go to work. ${ }^{6}$ A recent study conducted in quarantine facility centers in India reported that depression, anxiety, and stress were found in $49.4 \%, 40.9 \%$, and $75.8 \%$ of the residents, respectively. ${ }^{7}$

Quarantine, although designed to be protective of individual and public health, brings with it negative health impacts that need to be understood, prevented, and mitigated. The more public health officials understand the nature of the quarantine experience, the more it can be designed to reduce adverse impacts, promote protective factors, and optimize short and long-term outcomes from a mental and physical well-being perspective. This study contributes to the growing body of knowledge related to the impact of quarantine during widespread infectious diseases by exploring aspects of mental health at COVID-19 quarantine facilities.

Only a few studies have examined the degree of psychological impact on people experiencing involuntary quarantine inside an institutionalized facility, so the main objective of this study is to measure the effects of involuntary quarantine among individuals in quarantine-designated facilities during the COVID-19 pandemic in Saudi Arabia.

\section{Methodology \\ Study Design, Setting, and Sampling}

A cross-sectional study was conducted in order to assess the psychological responses of COVID-19 pandemic on individuals placed in institution-based quarantine facilities in the kingdom of Saudi Arabia. It was based on quarantined individuals in seven various hotels designated for quarantine purposes during the pandemic in Riyadh and Qassim regions of Saudi Arabia. Each facility constituted of covering medical doctors, nurses, social workers, and psychologists with number quarantined/isolated individuals ranged from 40 to 200 individuals in each facility; each individual was placed in a single room alone, reasons for quarantine ranged from being traveler returning to the kingdom to a confirmed cased with mild disease. Each individual was observed for vital signs at least twice a day, with necessary medication dispersion whenever there is a need by the medical doctor on duty. Individuals were quarantined for a planned period of 14 days, and the survey was distributed in the 8th-day quarantine period.

We used an online-based questionnaire provided by google survey platform (Google LLC, Mountain View, California, USA), the survey distributed to quarantined individuals through small cards with letters explaining the study purpose, objectives, time needed for completion, and inviting them to participate. A quick response barcode was provided in order to scan and participate in the study. Physical distribution was not feasible in order to minimize contact. All quarantined individuals in the selected facilities who fit inclusion criteria were invited to participate. Inclusion criteria were adult ( $>18$ years), Arabic speaker, Saudi citizen or resident of Saudi Arabia, 
and being quarantined or isolated in the facility. The study was conducted in accordance with the Declaration of Helsinki protocol and was approved by the Qassim Institutional Review Regional Ethical Committee (No.H04-Q-001). The study aims and objectives were explained to the respondents and they provided written informed consent. Data confidentiality was preserved throughout the study and was not breached unless for study needs. Data collected over a period from 29 April to19 May 2020, during a curfew was in place in the kingdom. We calculated sample size using Epi Info ${ }^{\mathrm{TM}} 7$ (Centers for Disease Control and Prevention (CDC), Atlanta, Georgia, USA). The calculation resulted in 192 respondents based on expected \% prevalence ( $\mathrm{p}$ ) of $14.6 \%$ of respondents reported a score $>20$ on the IES-R scale during SARS quarantine, $5 \%$ margin of error, and confidence interval (\%) of $95 \%{ }^{8}$

\section{Survey}

The study tool was adopted primarily from a study conducted during the early days of COVID-19 outbreak throughout China. ${ }^{9}$ The self-administered survey covered several area including the following: Sociodemographic such as age, gender, educational level, being married or not, if having children, employment or being a medical sector employee, income per household in Saudi Riyal (1 $\mathrm{USD}=3.75 \mathrm{SAR}$ ), housing status and residents' number in the house. The physical symptoms and health status as reported by quarantined individuals were also addressed (health status was rated from 1 to 5 , where higher number meant better health status). History of chronic medical or psychiatric disorders were also reported. Visiting a hospital, tested for COVID-19 or being admitted in the past 14 days were included. Additionally, respondents were asked to report the time spent in quarantine/isolation and reasons for their placement in quarantine or isolation.
The questionnaire included items related to COVID-19 knowledge and practices. These items included transmission routes, source of information, practiced preventive measures against SARS-Cov-2 such as wearing masks, hand hygiene, cough etiquette and others. In order to address concerns experienced by quarantined individuals, confidence of available diagnostic test for SARS-Cov-2, level of satisfaction of available health information and if they felt that there is unnecessary worrisome with regard to COVID-19 were covered. Participants were asked about their chances of being infected and survival probability if they contracted the infection. An additional question on the coping strategies practiced during the quarantine, including sleeping a lot, engaging in work, and others, were included.

To achieve study aim, the Impact of Event Scale-Revised (IES-R) and Depression, Anxiety, and Stress Scale (DASS21) were used. These scales were used in several studies assessing the psychological responses following other outbreaks such as SARS, MERS and COVID-19. ${ }^{9-11}$ The IES-R used to assess post traumatic symptoms experienced during the last week. It has been translated and validated in native Arabic speakers. ${ }^{12,13}$ This scale consists of 22 items, divided into three subscales mean avoidance, intrusion and hyperarousal. ${ }^{12}$ Quarantined individuals were asked to choose answers ranged from "not at all" to "extremely" (0 to 4). Scoring was applied as in Table $1 .^{12}$

To assess mental health status, Arabic version of 21 item DASS scale was used. ${ }^{14}$ This scale shown to be valid and reliable among Arabs. ${ }^{14}$ It consists of three subscales, namely depression, anxiety, and stress. Every single subscale provides a summary score for seven items. Responses to each item ranged from zero to three "did not apply to me" to "applied to me most of the time". Scoring for each subscale was different from one another as illustrated in Table $1 .^{15}$

Table I Scoring for Impact of Event Scale-Revised (IES-R) and Depression, Anxiety, and Stress Scale (DASS-2I)

\begin{tabular}{|l|l|l|l|l|}
\hline Interpretation & $\begin{array}{l}\text { Impact of Event Scale- } \\
\text { Revised (IES-R) }\end{array}$ & $\begin{array}{l}\text { Depression Items: 3, 5, 10, } \\
\text { I3, 16, I7, and 2I }\end{array}$ & $\begin{array}{l}\text { Anxiety Items: 2, 4, 7, 9, } \\
\text { 15, 19, and 20 }\end{array}$ & $\begin{array}{l}\text { Stress Items: I, 6, 8, II, } \\
\text { I2, 14, and I8 }\end{array}$ \\
\hline Normal & $0-23$ & $0-9$ & $0-6$ & $0-10$ \\
\hline Mild & $24-32$ & $10-12$ & $7-9$ & $11-18$ \\
\hline Moderate & $33-36$ & $13-20$ & $10-14$ & $19-26$ \\
\hline Severe & $>37$ & $21-27$ & $15-19$ & $27-34$ \\
\hline Extremely severe & NA & $28-42$ & $20-42$ & $35-42$ \\
\hline
\end{tabular}




\section{Statistical Analysis}

Data were collected from 214 participants and analyzed. Data were initially collected into a Microsoft Excel spreadsheet and later transferred into IBM SPSS Statistics for Windows, version 22.0 (IBM Corp., Armonk, N.Y., USA) for analysis. Descriptive analysis was carried out and the results presented in frequencies and percentages (for categorical data), and means and standard deviation (for continuous data). Chi-square tests (and Fischer's exact tests where appropriate) were carried out to identify statistically significant relationships between psychological health and each of sociodemographic factors, health status/service utilization factors, symptoms, knowledge about COVID-19, precautionary measures, and coping mechanisms. Psychological health was determined using the 22-item IES-R which assessed for subjective psychological distress, and the 21-item DASS with its three subscales assessing for stress, anxiety, and depression. Both scales were found to have strong reliability with Cronbach's alpha of 0.901 for the IES-R and 0.944 for the DASS. Similarly, the subscales of the DASS had strong reliability with each of the stress, anxiety, and depression subscales having Cronbach's alpha of $0.895,0.867$, and 0.858 , respectively. All tests were carried out at a level of significance of $\mathrm{p}<0.05$.

\section{Results}

\section{Psychological Impacts of COVID-19 Quarantine}

The average scores of the participants on the IES-R and the subscales of the DASS-21 were $23.3 \pm 14.7$ (IES-R), $9.3 \pm 10.7$ (DASS stress subscale), $4.8 \pm 8.1$ (DASS anxiety subscale), and $7.4 \pm 9.0$ (DASS depression subscale). The proportion of participants that met the criteria for stress, anxiety, and depression were $25.7 \%, 21.5 \%$, and $32.7 \%$, respectively. On the IES-R, $28.0 \%$ of the participants met the criteria for psychological distress (PTSD). It is important to note that the participants had varying degrees of severity of each of stress, anxiety, and depression, as summarized in Table 1.

\section{Sociodemographic Characteristics and Influence on Psychological Response to the COVID-19 Pandemic}

There were more males $(59.8 \%)$ than females in the study sample, with the majority of the participants being in the
18-30 years age category ( $56.1 \%)$ and closely followed by those in the $31-40$ years age category (30.4\%, Table 2). More than half of the population had at least a bachelor's degree $(41.1 \%)$ or a master's degree $(23.8 \%)$. The split across marital lines was almost similar, with $49.5 \%$ married and $49.1 \%$ single. Of the respondents who were currently or previously married, the majority had between 1 and 3 children (63.3\%). With regards to employment status, students constituted $45.3 \%$, while $39.3 \%$ others were in one form of employment or the other. As much as $10.7 \%$ of the participants were working in the medical field and $29.0 \%$ had at least a family member who works in the medical field.

Across the scales, the factors listed above were tested to identify which had a statistically significant relationship with stress, anxiety, and depression on the DASS and subjective psychological distress on the IES-R. On the IES-R, sex, age, number of children, and the number of individuals residing in the household had statistically significant influence on the prevalence of subjective psychological distress $(\mathrm{p}<0.05)$. Participants who were aged between 18 and 30 years $(p=0.039)$, females $(p=$ 0.014 ), were significantly more likely to have been distressed, as shown on the IES-R. Being female $(p=0.001)$, were found to be significantly associated with stress on the appropriate subscale of the DASS. Similarly, participants who are females $(p=0.027)$ are significantly more likely to suffer from anxiety.

Using the depression subscale of the DASS, those who were females $(p=0.041)$, within the age category of 18 to 30 years $(p=0.024)$, single $(p=0.022)$, having between 1 and 3 children $(p=0.003)$, and living with more than three people in the same household $(p=0.039)$ were significantly more likely to have depression. Factors such as educational level, employment status, family monthly income, working in the medical field, or having a family member that works in the medical field did not have any statistically significant relationship with subjective distress (on the IES-R), stress, anxiety, or depression on the DASS. (Supplementary Table 1 and 3)

\section{Health Status/Service Utilization and Symptoms Effect on Mental Health Status}

Looking at the relationships between these variables and participants expression of psychological distress, stress, anxiety, and depression (Table 3); all variables had a statistically significant relationship with one or more of 


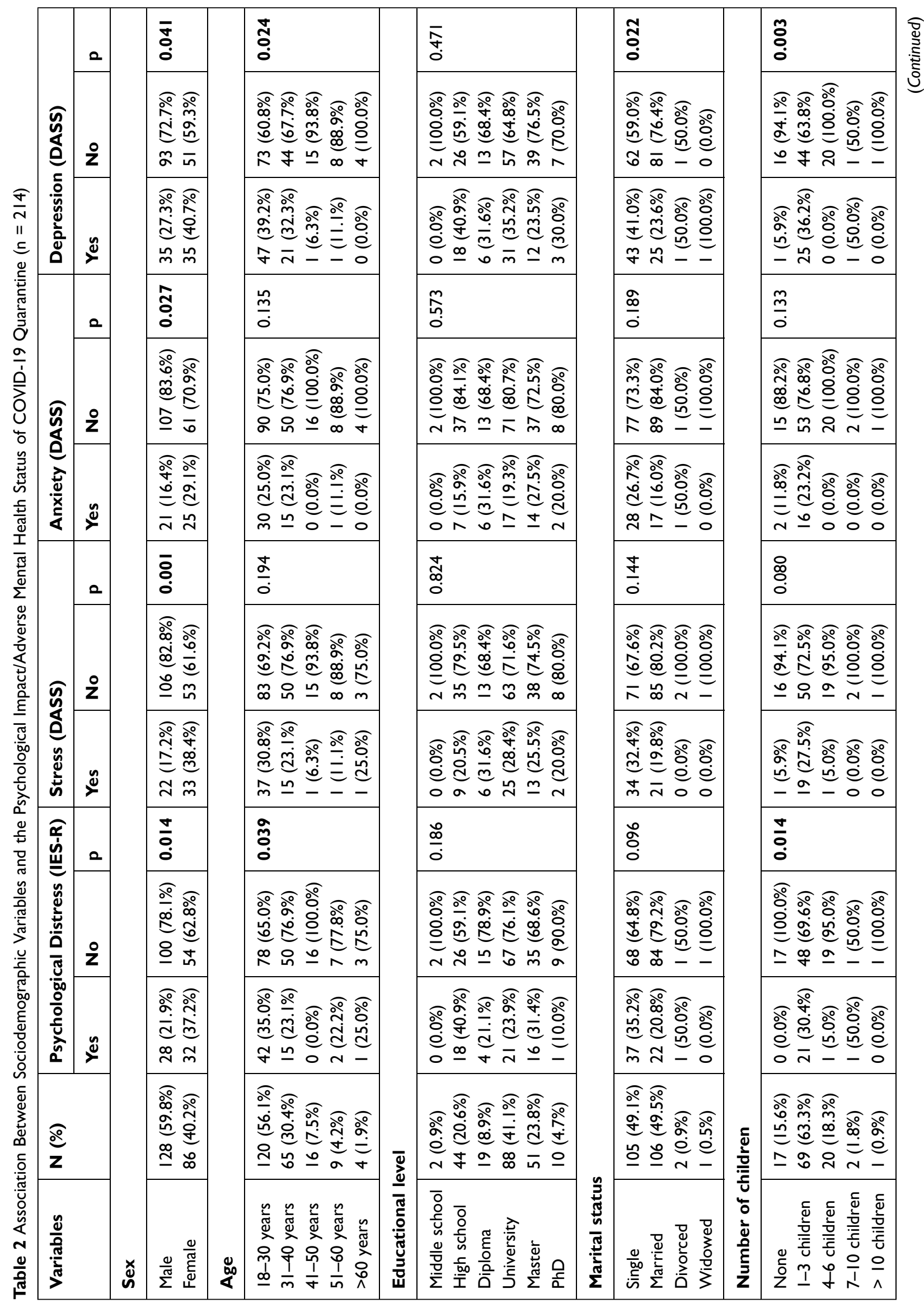




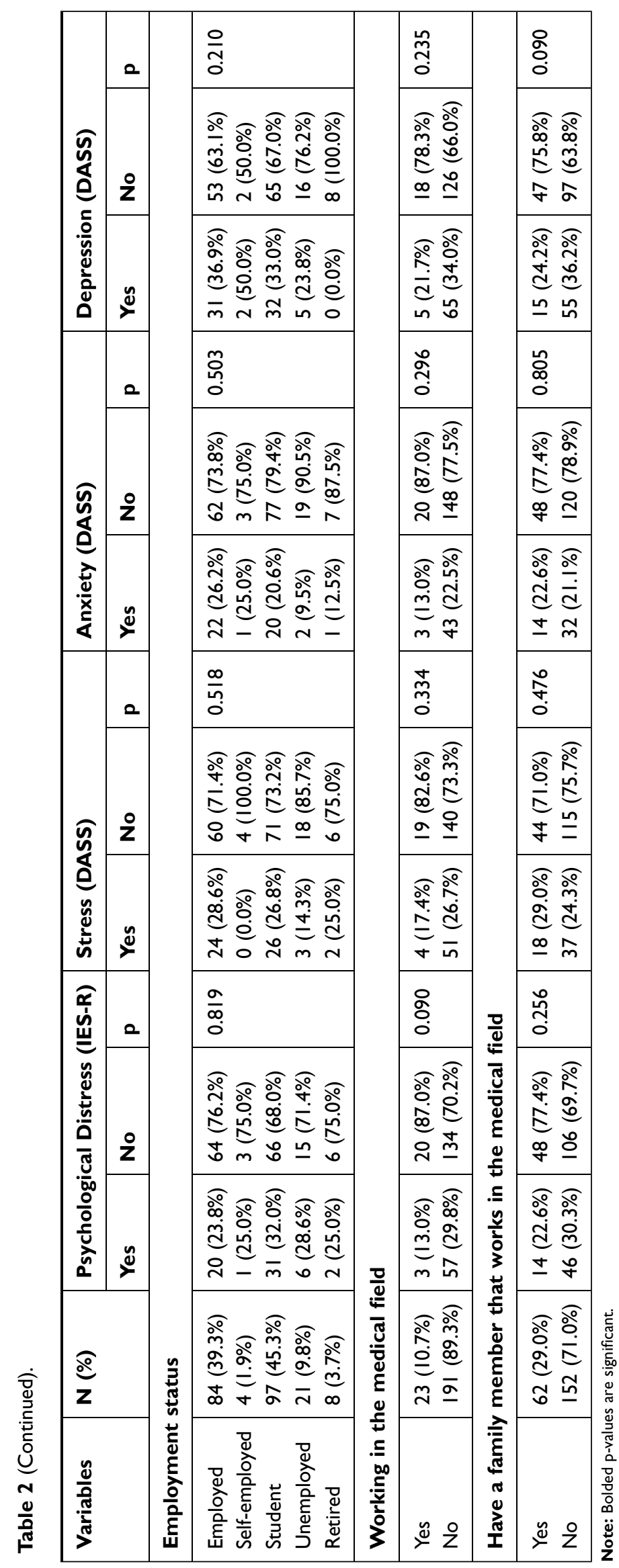




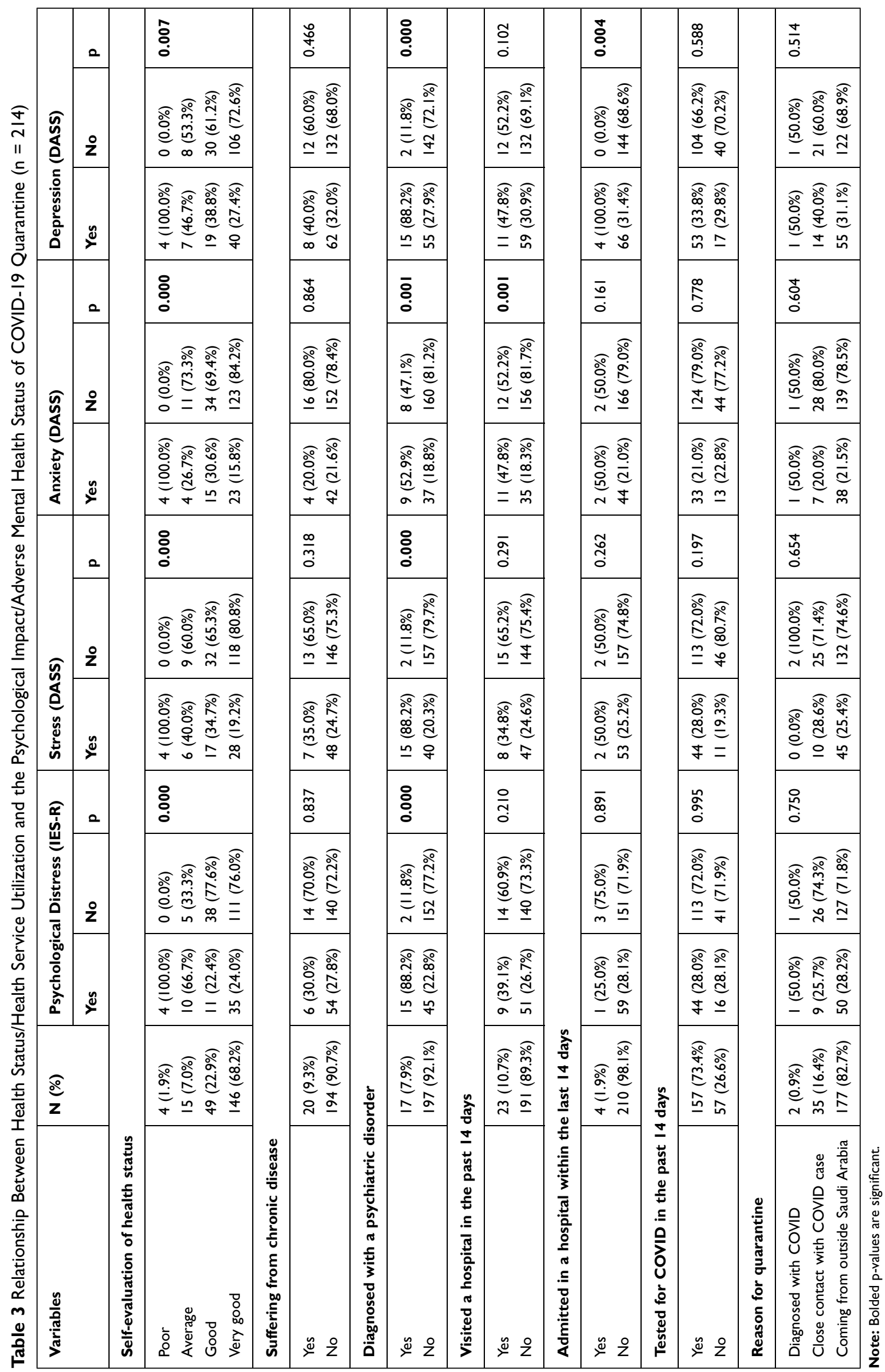




\begin{tabular}{|c|c|c|c|c|c|c|c|c|c|c|c|c|c|c|c|c|c|c|c|c|c|}
\hline & & a & & & $\begin{array}{l}\text { Ĩ } \\
\text { on }\end{array}$ & & $\begin{array}{l}\text { त్ } \\
\text { o }\end{array}$ & & : & & $\underset{\widetilde{\pi}}{\tilde{O}}$ & & $\begin{array}{l}\stackrel{0}{0} \\
0 \\
0\end{array}$ & & $\frac{\stackrel{\sigma}{o}}{0}$ & & $\frac{\bar{n}}{0}$ & & 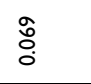 & & 念 \\
\hline & $\begin{array}{l}\bar{y} \\
\text { } \\
\text { }\end{array}$ & $\stackrel{\circ}{z}$ & & & 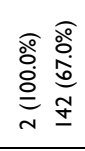 & & 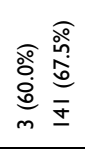 & & 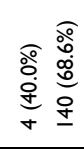 & & 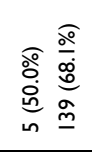 & & 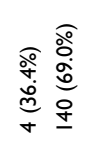 & & 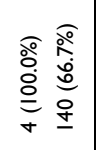 & & 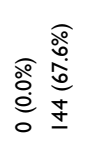 & & 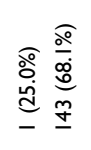 & & 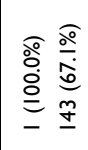 \\
\hline & 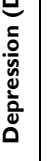 & $\stackrel{\check{x}}{\nu}$ & & & 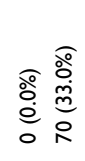 & & 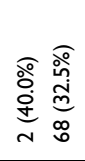 & & 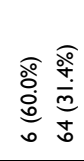 & & 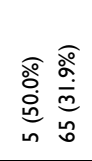 & & 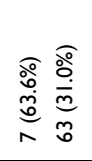 & & 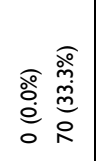 & & 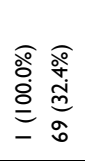 & & 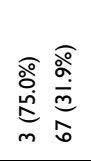 & & 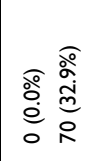 \\
\hline & & a & & & 占 & & $\bar{o}$ & & 㟔 & & $\stackrel{\text { ֻ̆ }}{0}$ & & $\stackrel{0}{0}$ & & : & & $\begin{array}{l}\text { 员 } \\
\text { o. }\end{array}$ & & స్ & & : \\
\hline & 勿 & $\stackrel{0}{z}$ & & & 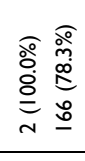 & & 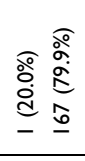 & & 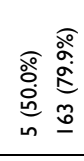 & & 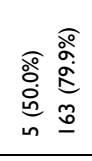 & & 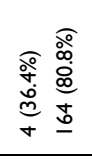 & & 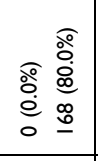 & & 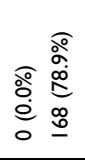 & & 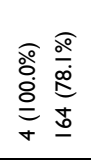 & & 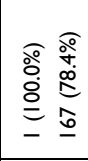 \\
\hline & 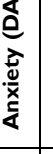 & $\stackrel{\Xi}{\nu}$ & & & 总 & & 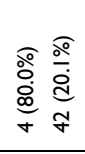 & & 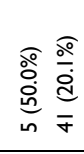 & & 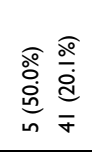 & & 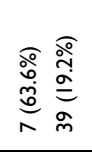 & & 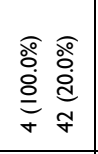 & & 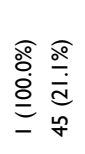 & & 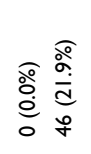 & & 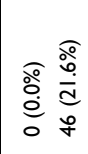 \\
\hline & & a & & & $\begin{array}{l}\text { ợ } \\
\text { on }\end{array}$ & & $\begin{array}{l}\stackrel{0}{0} \\
\text { Oे }\end{array}$ & & $\begin{array}{l}\text { Ô. } \\
\text { Oे }\end{array}$ & & 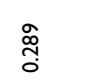 & & $\frac{\text { I }}{0}$ & & $\underset{0}{\tilde{0}}$ & & 瓷 & & 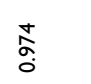 & & 㔛 \\
\hline & & $\stackrel{0}{z}$ & & & 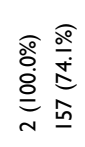 & & 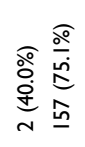 & & 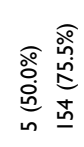 & & 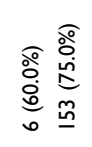 & & 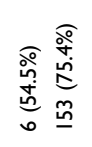 & & 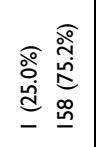 & & 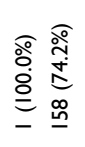 & & 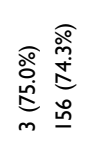 & & 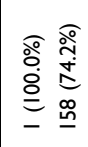 \\
\hline & 产 & $\stackrel{y}{\check{\nu}}$ & & & 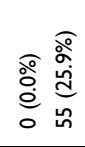 & & 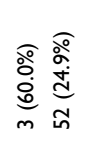 & & 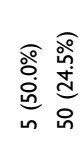 & & 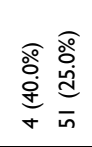 & & 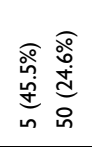 & & 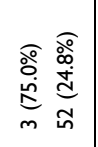 & & 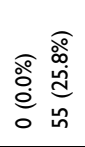 & & 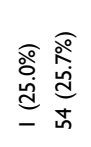 & & 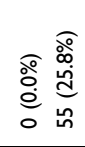 \\
\hline & & 2 & & & $\begin{array}{l}\stackrel{n}{\hat{m}} \\
0\end{array}$ & & $\frac{\hat{o}}{0}$ & & $\begin{array}{l}\bar{o} \\
\text { Oे }\end{array}$ & & $\frac{m}{0}$ & & $\frac{\hat{\infty}}{0}$ & & $\begin{array}{l}\stackrel{\tilde{O}}{0} \\
\stackrel{0}{0}\end{array}$ & & 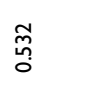 & & 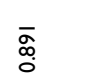 & & 雚 \\
\hline & & ż & & & 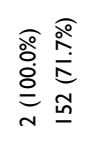 & & 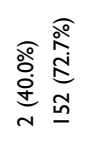 & & 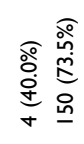 & & 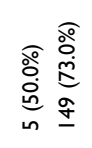 & & 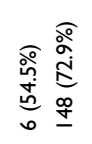 & & 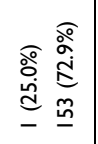 & & 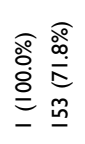 & & 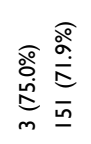 & & 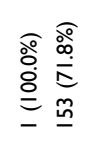 \\
\hline & 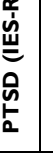 & $\stackrel{\check{\nu}}{\check{\nu}}$ & & & 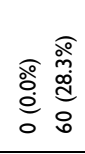 & & 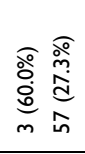 & & 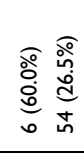 & & 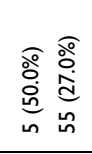 & & 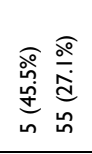 & & 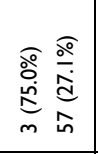 & & 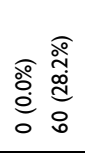 & & 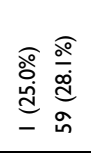 & & 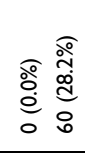 \\
\hline & $\begin{array}{l}\text { ¿̊ } \\
z\end{array}$ & & & & 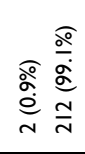 & & 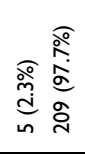 & & 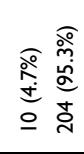 & & 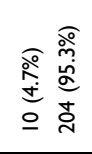 & & 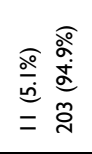 & & 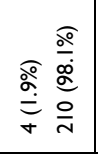 & & 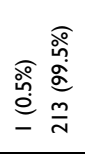 & & 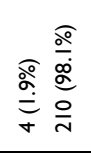 & & 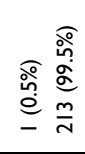 \\
\hline & 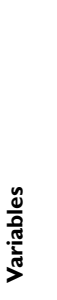 & & 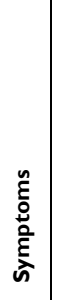 & $\begin{array}{l}\text { 总 } \\
\text { uे }\end{array}$ & $\stackrel{\mathscr{g}}{\longleftarrow} \stackrel{0}{z}$ & 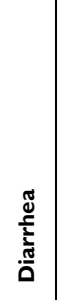 & 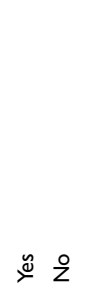 & 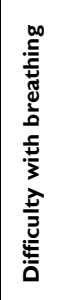 & $\stackrel{\mathscr{c}}{*} \frac{0}{z}$ & 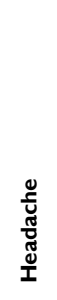 & $\underbrace{\mathscr{c}}_{\mathscr{c}} \frac{0}{z}$ & 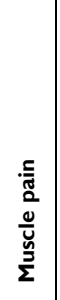 & 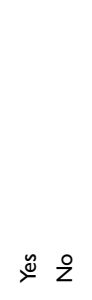 & 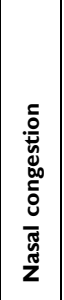 & $\stackrel{\mathscr{x}}{\longleftarrow} \stackrel{0}{z}$ & 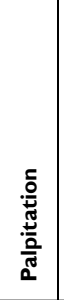 & 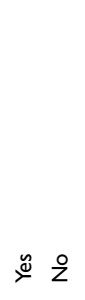 & 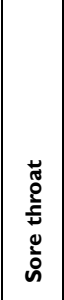 & 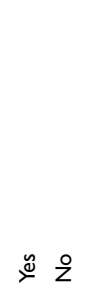 & 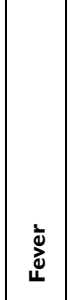 & 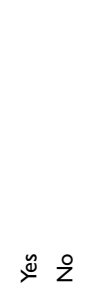 \\
\hline
\end{tabular}




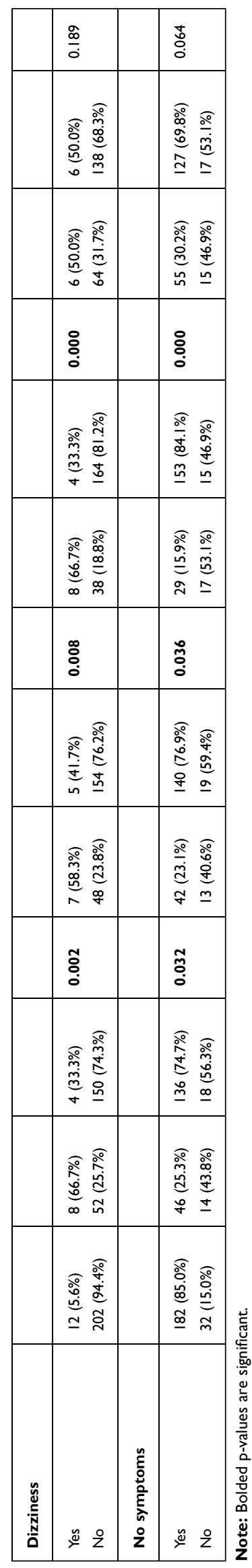

psychological distress, stress, anxiety, and depression ( $\mathrm{p}<$ 0.05 ) except for having being diagnosed with a chronic disease, being tested for COVID-19 in the preceding 14 days, and reasons for the quarantine. On the IES-R, participants who noted that they had average health $(\mathrm{p}<0.001)$ and those who had previously been diagnosed with a psychiatric disorder $(p<0.001)$ were significantly more likely to have psychological distress than their counterparts. On the stress subscale of the DASS, participants who believe their health status is average $(\mathrm{p}<0.001)$, and have been previously diagnosed with a mental health disorder $(\mathrm{p}<0.001)$ were significantly more likely to exhibit stress. Similarly, anxiety was significantly associated with being of good to average health $(p<0.001)$, previous diagnosis with a psychiatric disorder $(\mathrm{p}=0.001)$, and visiting a hospital in the preceding 14 days (as assessed using the anxiety subscale of the DASS; $p=$ $0.001)$.

Depression also appeared to be strongly associated with similar factors. Participants who were of average health $(\mathrm{p}=0.007)$, been previously diagnosed with a psychiatric disorder $(\mathrm{p}<0.001)$, and/or admitted in a hospital in the last 14 days $(\mathrm{p}=0.004)$ had a significantly increased risk of depression (DASS-Depression subscale).

Participants were asked questions relating to symptoms they had experienced in the few days preceding the study (Table 4), the most predominant symptoms experienced were dizziness $(5.6 \%)$, muscle pain $(5.1 \%)$.

\section{Knowledge About COVID-I9 and Psychological Impact}

On the relationship between knowledge/concerns about COVID-19 and the psychological impact/adverse mental health status of COVID-19 quarantine (Table 5), the majority of the participants correctly indicated that coronavirus was transmitted via exposure to droplets from sneezing and coughing and direct contact with infected surfaces $(94.4 \%)$. However, this knowledge had no significant impact on whether they had psychological distress, stress, anxiety, and depression. The participants had strong confidence in the available options of diagnosing the disease, with $86.0 \%$ of them ranking high to very high on their confidence level. This had only a significant impact on stress and depression as those who had average confidence levels were more likely to suffer from stress $(\mathrm{p}=$ $0.014)$ and/or depression $(p=0.002)$ compared with their counterparts with stronger confidence levels. On 


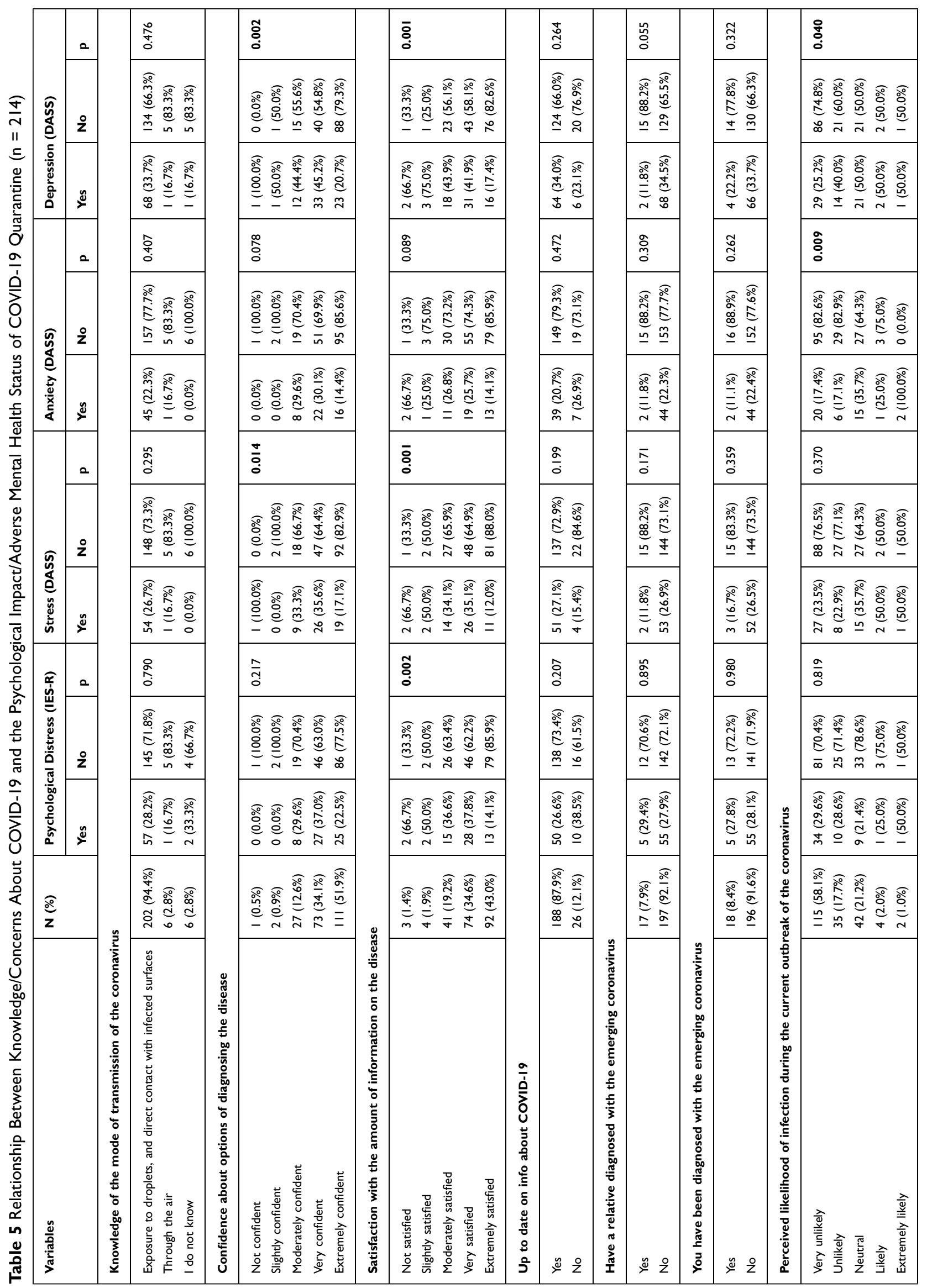




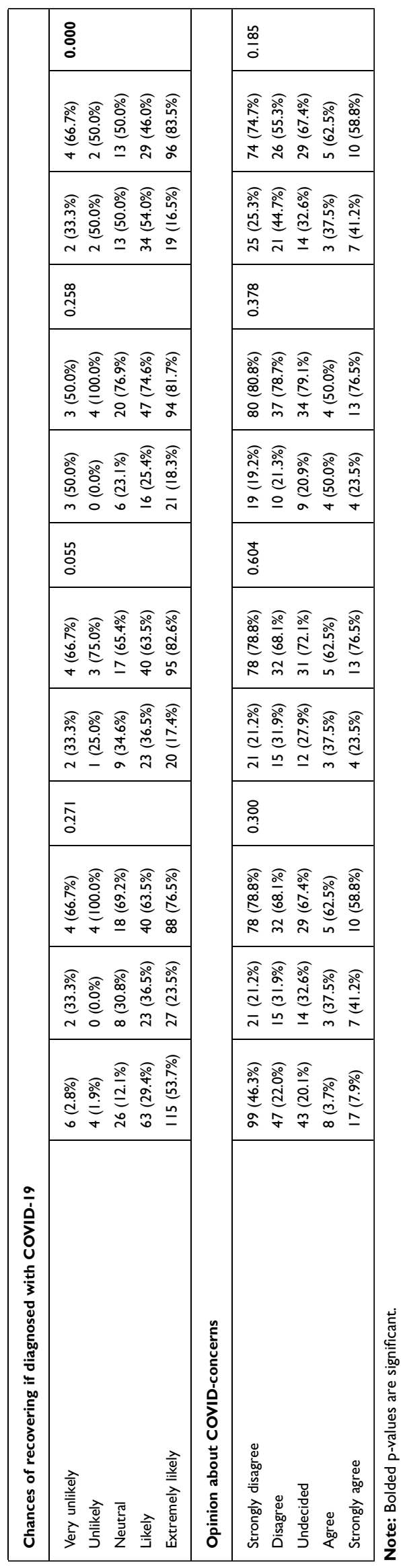

satisfaction with the amount of information available about the disease, $77.6 \%$ of the participants were satisfied or very satisfied. This factor had a statistically significant influence on psychological distress, stress, and depression as those who were averagely satisfied with the amount of information available on COVID-19 were more likely to have psychological distress $(p=0.002)$, stress $(p=0.001)$, and/or depression $(p=0.001)$ compared with those who were very satisfied. Being up to date on information about COVID-19 by $87.9 \%$ of the participants had no significant influence on psychological distress, stress, anxiety, or depression.

Up to $7.9 \%$ of the participants had relatives diagnosed with the coronavirus, but this did not significantly influence their psychological health. However, $8.4 \%$ of the participants had been diagnosed with COVID-19, and interestingly, this was not found to have any statistically significant relationship with either psychological distress, stress, anxiety, or depression. With regards to the perceived likelihood of infection during the current outbreak, the majority of the participants believed that they were unlikely to be infected and this factor had significant influence on their psychological health as those who thought they were likely to get infected had higher chances of having anxiety $(\mathrm{p}=0.009)$ and/or depression $(\mathrm{p}=$ 0.040). Similarly, the majority believed that even if they became infected, their chances of recovering were very high. Participants who did not know what their chances of recovering or who thought they were unlikely to recover were more likely to have depression $(\mathrm{p}<0.001)$. (supple mentary Table 2)

\section{Precautionary Measures and Psychological Impact}

Participants in the study had been using various means to protect themselves from getting infected with the coronavirus (Table 6). What the majority of them have been doing include washing and disinfecting hands (96.3\%), avoiding handshakes (87.4\%), using face masks $(85.0 \%)$, social distancing $(84.6 \%)$, and avoiding the sharing of utensils during meals (57.0\%).

Regarding the relationships between these protective measures and psychological health, only the use of a face mask had any significant influence on the expression of psychological distress. Participants who used face masks were significantly more likely to have psychological distress compared with those who did not $(p=0.020)$. 


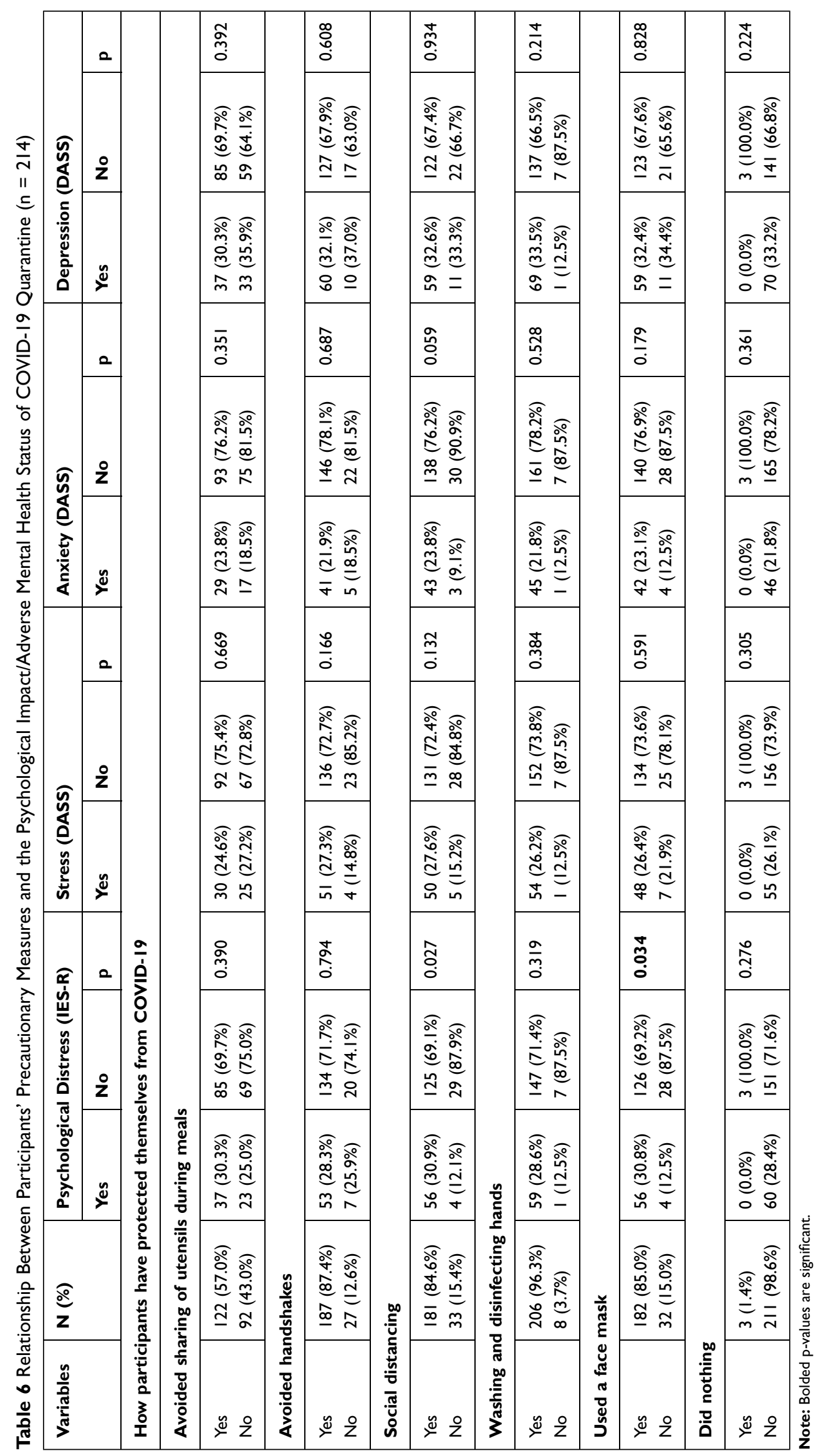




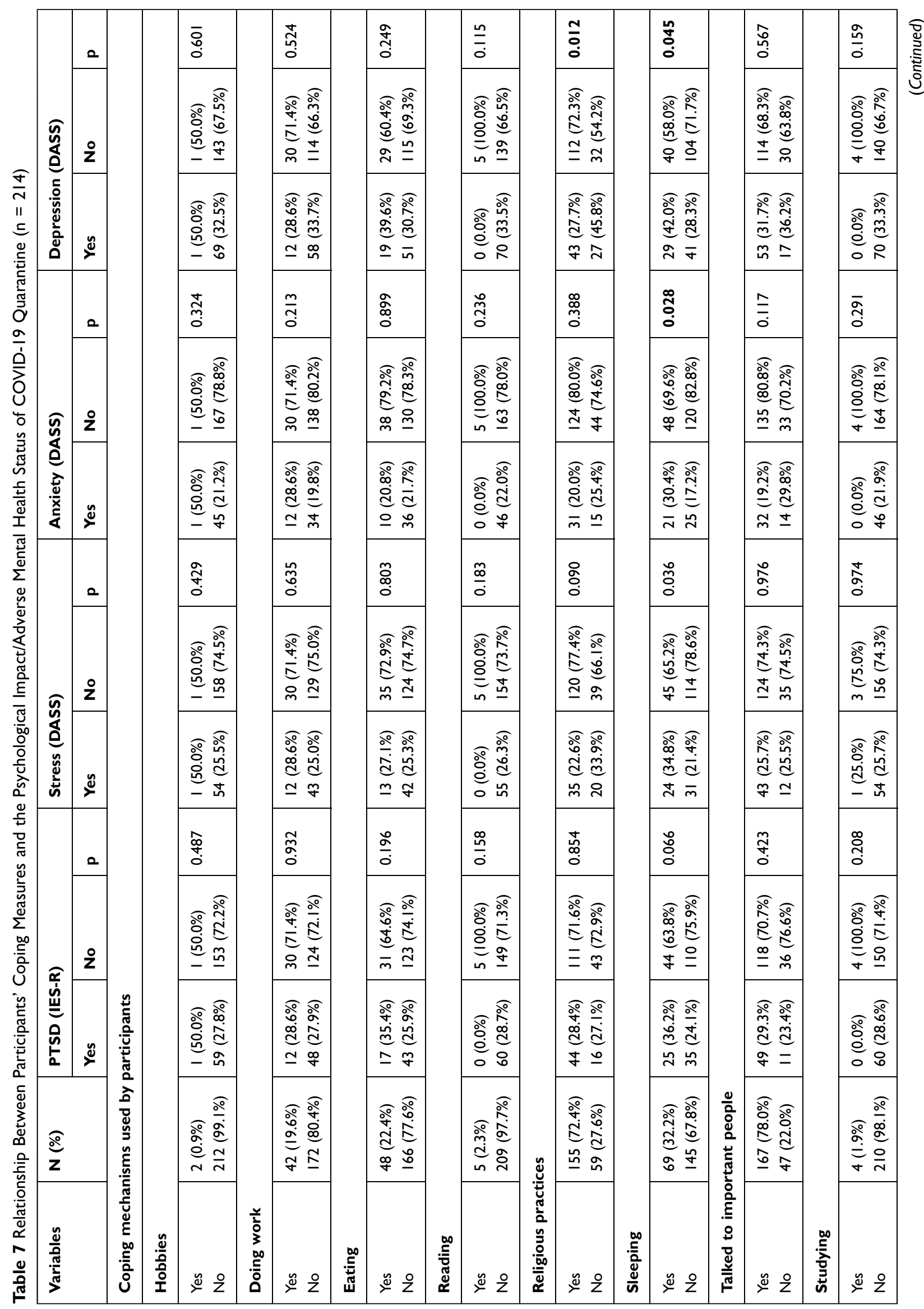




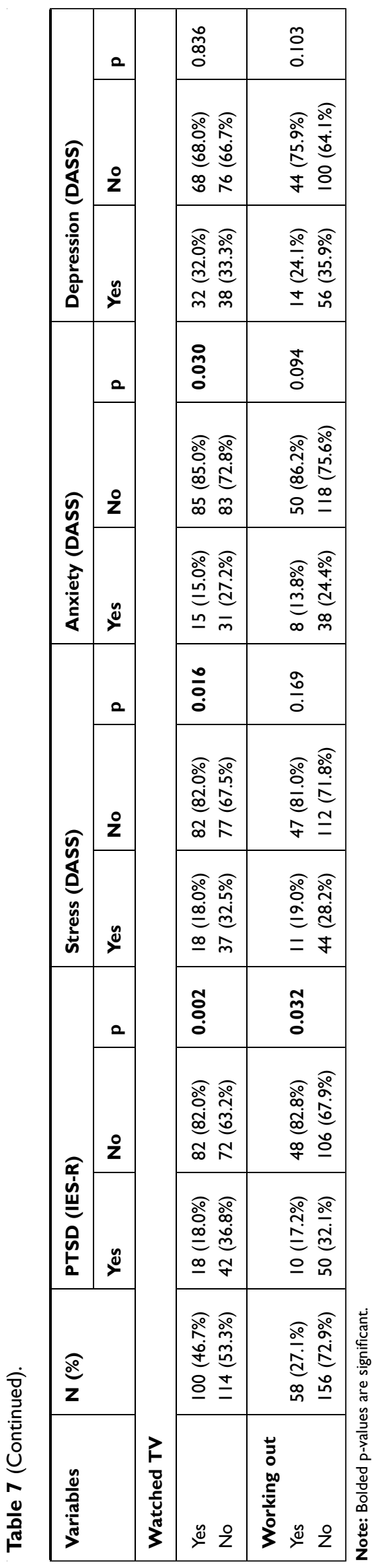

\section{Coping Mechanisms and Psychological Impact}

A wide variety of coping mechanisms have been in use by participants to manage the stress associated with the COVID-19 quarantine (Table 7). The wide array of coping measures includes talking to important people in their lives (78.0\%), engaging in religious practices $(72.4 \%)$, watching TV (46.7\%), sleeping (32.2\%), working out (27.1\%), eating (22.4\%), and doing work-related tasks (19.6\%). Other less commonly used coping measures include reading $(2.3 \%)$, studying $(1.9 \%)$, and participating in hobbies $(0.9 \%)$. Some of these coping mechanisms significantly improved or worsened the psychological health of the participants. Using sleep as a coping mechanism increased the risk of anxiety $(\mathrm{p}=0.028)$ and/or depression $(\mathrm{p}=$ 0.045). On the other hand, participants who did not participate in religious practices were more likely to have depression $(\mathrm{p}=0.012)$; same as those who did not watch TV who were more likely to have psychological distress ( $p$ $=0.002)$, stress $(p=0.016)$, and $/$ or anxiety $(p=0.030)$. Working out was also found to be protective as those who chose not to were more likely to suffer from psychological distress $(\mathrm{p}=0.032)$.

\section{Discussion}

The findings of this study contribute to the growing body of knowledge related to the impact of quarantine during widespread infectious diseases by exploring aspects of mental health during a COVID-19 quarantine. This study helps respond to the call for action put forth by Holmes et al. $2020 .^{16}$

The rates of mental health symptoms found in this study are consistent with the observation that quarantine and lockdowns have a psychological impact. Psychological distress, depression, anxiety, insomnia, stigma, and low self-esteem have all been associated with quarantine. ${ }^{17}$ These associations provide a compelling argument for the establishment and implementation of protective measures in anticipation of both COVID-19's "second wave" and future infectious outbreaks.

This is one of the few studies that assesses the psychological impact of involuntary quarantine during the COVID-19 pandemic. The participants met the criteria for stress, anxiety, and depression at a rate of $25.7 \%$, $21.5 \%$, and $32.7 \%$, respectively, while $28.0 \%$ reported the psychological impact of quarantine as moderate or severe. These findings are consistent with a recent study 
which reported participants' level of anxiety and depression to be $22.2 \%$ and $22.1 \%$, respectively, for people under hotel quarantine. ${ }^{18}$ Another study conducted during an equine influenza outbreak in Australia found that $34 \%$ of quarantined horse owners reported psychological distress. ${ }^{19}$

An increased rate of psychological distress was found in single females. This finding is in keeping with other studies demonstrating that demographic factors can influence psychological responses to an infectious outbreak in the community. Taylor et al found that younger people were more at risk of psychological distress during Australia's epidemic of equine flu. ${ }^{19}$ Guo et al found that female sex predicted higher perceived helplessness scores in a study of quarantined COVID-19 patients. $^{9}$ Park et al reported that young age, female gender, and caregiver status were linked with increased stressor exposure and stressfulness during the COVID-19 pandemic. $^{20}$ Younger orthopedic surgeons experienced more stress during a COVID-19 lockdown in India than older orthopedic surgeons. ${ }^{21}$

This study did not identify health-care worker status as being a risk factor for increased mental health symptoms in response to quarantine. However, many authors have documented the negative psychological sequelae of quarantine or lockdown on health-care providers. ${ }^{21-23}$

This study found an association between face mask use and psychological distress symptoms. As a tangible and visible reminder of the infectious outbreak, a face mask may act as a trigger for psychological distress. Likewise, a mask may invoke more stigma than washing hands or social distancing. Stigma has been identified as a source of psychological distress in several studies. ${ }^{9,24-26}$

A history of reported psychiatric disorder and selfreported poor health was predominant across all scales. This is consistent with findings during the MERS outbreak in South Korea that reported that stressors like quarantine or isolation might worsen the pre-existing mental illnesses. $^{25,27}$ Self-reported poor health is known to be associated with signs of psychological distress; this study's results demonstrate that respondents with poor/average self-reported health status report greater psychological distress, depression, anxiety, and stress symptoms than their counterparts. ${ }^{28}$ The association between physical and mental symptoms observed in this study is not specific to the quarantine or COVID-19. This relationship is well documented in the literature. ${ }^{29-32}$ In this study, more somatic symptoms were associated with anxiety than with stress, psychological distress, or depression. This finding may reflect an acute, fear-based response to experiencing symptoms indicative of a COVID-19 infection. Participants in this study who felt more likely to get infected with COVID-19 had an elevated risk of anxiety symptoms. This result is not unlike the reported association between perceived risk of infection and depression during the SARS epidemic. ${ }^{26}$

A diagnosis of COVID-19 did not result in higher rates of psychological distress, depression, anxiety, or stress. It may be that for some people the possibility of getting COVID-19 and the associated unknowns invoke more distress than the lived experience. Both unknowns and anticipation of a possible threat have been linked to anxiety. ${ }^{33}$ Lower rates of satisfaction with COVID-related information increased the likelihood of psychological distress, stress, and depression. Others have reported the negative impact of suboptimal information provision during outbreaks. ${ }^{22}$ Brooks et al $2020^{34}$ noted that communication about quarantine rationale and requirements is vital to reducing the psychological stress of isolation. Findings like these have resulted in strong advocacy for providing timely and accurate communication to those significantly impacted by infectious outbreaks. ${ }^{25,35,36}$ Sahu et $\mathrm{al}^{21}$ recommended that open communication be prioritized to alleviate some of the stress experienced by doctors during the lockdown. Internet-based interventions during quarantine offer the opportunity to mitigate the distress of participants by addressing "unknowns." Relevant and potentially reassuring information could be provided in concert with the psychological support provided by psychoeducation, coping skills, and mindfulness training. ${ }^{37}$

The level of engagement with precautionary measures in this study was high, and the percentage of complete nonparticipation low. However, even low rates of noncompliance can sabotage quarantine efforts and lower the beneficial impact of cooperation by others. High levels of targeted information about quarantine and the infectious outbreak may help lower the number of individuals who wholly or partially reject recommended safety measures.${ }^{8,38}$ Additionally, compliance with the protective measures recommended by health authorities has been shown to be varied by the perceived benefit (or efficacy) of that recommended measure. This variation may also be influenced by one's self-efficacy, ie one's belief in their ability to accomplish a measure or action. Perceived risk of infection and one's belief that they can make a difference in the community may influence that relationship and thereby influence the level of compliance practiced by the individuals. $^{39}$ 
This study contributes to the understanding of coping mechanisms used during quarantine and their ability to provide psychological benefits. The exploration of this topic remains a priority for those working towards minimizing the negative impact of quarantine on well-being. ${ }^{40}$ The study's results demonstrated a protective effect of religious activities against depression. Many religious practices include the concept of altruism or the "greater good." Lui et al showed that an "altruistic acceptance of risk" was protective against depressive symptoms in those quarantined during the SARS outbreak. Explicitly encouraging altruistic behavior during quarantine may prove beneficial. ${ }^{38}$ Sleep can be an avoidance behavior. The link between avoidance and anxiety and depression41,42 may explain why some mental health symptoms were higher in those using sleep to manage quarantine-related stress. Park et al found distractions to be commonly used as a coping mechanism by American workers during the COVID-19 pandemic. Watching television and working out are distractions that provided some psychological benefits in this study. ${ }^{20}$ As physical activity can decrease during quarantine, ${ }^{43}$ overtly facilitating this coping mechanism through such avenues as online exercise programs may be beneficial. Others have suggested that in quarantine some people cope with stress by eating and drinking in an attempt to feel better (also known as "stress-related eating"). ${ }^{44}$

Internal resources may not be sufficient to offset the psychological impact of living through a pandemic and being quarantined. In response, Kang et $\mathrm{al}^{45}$ reported on an initiative that provided cognitive behavioral therapy, progressive muscular relaxation, mindfulness, relevant education, online self-help programs, and telephone crisis lines to address the mental distress associated with the COVID19 quarantine. Telemedicine provides a venue to address deficits in adaptive, effective coping mechanisms within a quarantined or pandemic-affected population. ${ }^{37}$ Bäuerle et al and Balanzá-Martínez et al advocated for online health resources to address the mental health needs of the population during infectious outbreaks. ${ }^{40}$

This study shows increased levels of psychological distress, depression, anxiety, and stress symptoms among individuals in a quarantine-designated facility, indicating the importance of providing proper psychological support for those individuals, with more focus on vulnerable groups with a history of psychiatric illnesses or poor health status. It is also important to educate quarantined individuals with appropriate ways to cope with stress, such as working out or praying, and to provide proper tools to help them do that in the confined space of quarantine, if possible.

One of the study's limitations is the cross-sectional design, which provides only a transient view of quarantined individuals' psychological responses. Longitudinal studies might be needed to evaluate whether those responses will last after the quarantine/isolation is discontinued. Furthermore, this study utilized self-reported scales for the history of chronic diseases, psychiatric illnesses, and psychiatric symptoms, which were not confirmed by reviewing medical records or medical or psychiatric interviews; self-reporting may have limited reliability when compared to interviews conducted by a specialist. To minimize contact, researchers approached the respondents through cards with printed QR codes; this may have prevented some individuals from participating if they were unfamiliar with such a method. The study included seven selected facilities/hotels in two regions of the kingdoms, which might limit the generalizability of the study's results to other regions or facilities run by institutions other than the ministry of health in the kingdom; however, this study provides an insight into the psychological impact of involuntarily quarantined individuals in designated facilities which are rarely reported in the literature.

\section{Conclusions}

In conclusion, during an institutional involuntary quarantine imposed by the government of Saudi Arabia, nearly one-fourth of the sampled population experienced a moderate or severe psychological impact. Females and single individuals had an increased rate of psychological distress. This study found that religious activities and exercise had a protective effect against depression and psychological distress. This study contributes to the ongoing conversation about the psychological aspects of being quarantined but much work remains to be done. The results of this study call not only for more research but also for the timely implementation of measures to prevent and mitigate the psychological distress seen in those living through the quarantine experience in designated facilities.

\section{Acknowledgments}

We would like to extend our thanks to all respondents and participants. Researchers would like to thank the Deanship of Scientific Research, Qassim University, for funding publication of this project. 


\section{Author Contributions}

Conceptualization, Abdulmajeed Alkhamees and Moath Aljohani; Data curation, Moath Aljohani; Formal analysis, Abdulmajeed Alkhamees; Investigation, Abdulmajeed Alkhamees; Methodology, Abdulmajeed Alkhamees, Ali T. Alhabib, Mohammed A ALGHESEN and Moath Aljohani; Project administration, Abdulmajeed Alkhamees; Supervision, Abdulmajeed Alkhamees and Moath Aljohani; Writing - original draft, Abdulmajeed Alkhamees, Ali T. Alhabib, Mohammed A ALGHESEN and Moath Aljohani; Writing - review \& editing, Abdulmajeed Alkhamees, Ali T. Alhabib, Mohammed A ALGHESEN and Moath Aljohani. All authors made substantial contributions to conception and design, acquisition of data, or analysis and interpretation of data; took part in drafting the article or revising it critically for important intellectual content; agreed to submit to the current journal; gave final approval of the version to be published; and agree to be accountable for all aspects of the work.

\section{Funding}

This research did not receive any specific grant from funding agencies in the public, commercial, or not-forprofit sectors.

\section{Disclosure}

The authors declare no conflicts of interest.

\section{References}

1. Most countries in the world have imposed partial or complete border closures to foreign nationals due to coronavirus outbreak | pew research center [Internet]. [cited April 19, 2020]. Available from: https://www. pewresearch.org/fact-tank/2020/04/01/more-than-nine-in-ten-peopleworldwide-live-in-countries-with-travel-restrictions-amid-covid-19/ft 2020-04-01_borderclosures/. Accessed December 9, 2020.

2. Coronavirus (COVID-19) events as they happen [Internet]. [cited April 19, 2020]. Available from: https://www.who.int/emergencies/dis eases/novel-coronavirus-2019/events-as-they-happen. Accessed December 9, 2020

3. Oh Don M. The Korean middle east respiratory syndrome coronavirus outbreak and our responsibility to the global scientific community. Infect Chemother. 2016;48:145-146.

4. Krumkamp R, Duerr HP, Reintjes R, Ahmad A, Kassen A, Eichner M. Impact of public health interventions in controlling the spread of SARS: modelling of intervention scenarios. Int $J$ Hyg Environ Health. 2009;212(1):67-75. doi:10.1016/j.ijheh.2008.01.004

5. Bai YM, Lin CC, Lin CY, Chen JY, Chue CM, Chou P. Survey of stress reactions among health care workers involved with the SARS outbreak. Psychiatric Ser. 2004;55(9):1055-1057. doi:10.1176/appi. ps.55.9.1055

6. Mazza C, Ricci E, Biondi S, et al. A nationwide survey of psychological distress among italian people during the COVID-19 pandemic: immediate psychological responses and associated factors. Int $J$ Environ Res Public Health. 2020;17(9):3165. doi:10.3390/ ijerph17093165
7. Upadhyay R, Sweta SB, Singh U. Psychological impact of quarantine period on asymptomatic individuals with COVID-19. Soc Sci Humanit Open. 2020;2(1):100061.

8. Reynolds DL, Garay JR, Deamond SL, Moran MK, Gold W, Styra R. Understanding, compliance and psychological impact of the SARS quarantine experience. Epidemiol Infect. 2008;136(7):997-1007. doi:10.1017/S0950268807009156

9. Guo Q, Zheng Y, Shi J, et al. Immediate psychological distress in quarantined patients with COVID-19 and its association with peripheral inflammation: a mixed-method study. Brain Behav Immun. 2020

10. Lee SM, Kang WS, Cho AR, Kim T, Park JK. Psychological impact of the 2015 MERS outbreak on hospital workers and quarantined hemodialysis patients. Compr Psychiatry. 2018;87:123-127. doi:10.1016/j.comppsych.2018.10.003

11. McAlonan GM, Lee AM, Cheung V, et al. Immediate and sustained psychological impact of an emerging infectious disease outbreak on health care workers. Can J Psychiatry. 2007;52(4):241-247. doi: $10.1177 / 070674370705200406$

12. Beck JG, Grant DMM, Read JP, et al. The impact of event scalerevised: psychometric properties in a sample of motor vehicle accident survivors. J Anxiety Disord. 2008;22(2):187-198. doi:10.1016/j. janxdis.2007.02.007

13. Davey C, Heard R, Lennings C. Development of the Arabic versions of the impact of events scale-revised and the posttraumatic growth inventory to assess trauma and growth in middle eastern refugees in Australia. Clin Psychol. 2015;19(3):131-139. doi:10.1111/cp.12043

14. Ali AM, Ahmed A, Sharaf A, Kawakami N, Abdeldayem SM, Green J. The Arabic version of the depression anxiety stress scale-21: cumulative scaling and discriminant-validation testing. Asian $J$ Psychiatr. 2017;30:56-58. doi:10.1016/j.ajp.2017.07.018

15. Gloster AT, Rhoades HM, Novy D, et al. Psychometric properties of the depression anxiety and stress scale- 21 in older primary care patients. $J$ Affect Disord. 2008;110(3):248-259. doi:10.1016/j. jad.2008.01.023

16. Holmes EA, O'Connor RC, Perry VH, et al. Multidisciplinary research priorities for the COVID-19 pandemic: a call for action for mental health science. Lancet Psychiatry. 2020;7(6):547-560. doi:10.1016/S2215-0366(20)30168-1

17. Hossain MM, Sultana A, Purohit N. Mental health outcomes of quarantine and isolation for infection prevention: a systematic umbrella review of the global evidence. Epidemiol Health. 2020; e2020038. doi:10.4178/epih.e2020038

18. Zhu S, Wu Y, Zhu C, Hong W, Yu Z, Chen Z. The Immediate Mental Health Impacts of the COVID-19 Pandemic Among People with or Without Quarantine Managements. Brain, Behavior, and Immunity. Academic Press Inc; 2020.

19. Taylor MR, Agho KE, Stevens GJ, Raphael B. Factors influencing psychological distress during a disease epidemic: data from Australia's first outbreak of equine influenza. BMC Public Health. 2008;8(1):347. doi:10.1186/1471-2458-8-347

20. Park CL, Russell BS, Fendrich M, Finkelstein-Fox L, Hutchison M, Becker J. Americans' COVID-19 stress, coping, and adherence to CDC guidelines. $J$ Gen Intern Med. 2020;1-8.

21. Sahu D, Agrawal T, Rathod V, Bagaria V. Impact of COVID 19 lockdown on orthopaedic surgeons in India: a survey. J Clin Orthop Trauma. 2020;11(Suppl 3):S283-90. doi:10.1016/j.jcot.2020.05.007

22. Fawaz M, Samaha A. The psychosocial effects of being quarantined following exposure to COVID-19: a qualitative study of Lebanese health care workers. Int J Soc Psychiatry. 2020;2020:002076402093220.

23. Wynn F, Peter E. Editorial. Nurs Inq. 2003;10(4):207-208. doi:10.1046/j.1440-1800.2003.00194.x

24. Morioka S, Saito S, Hayakawa K, et al. Psychiatric burdens or stress during hospitalization and concerns after discharge in patients with severe acute respiratory syndrome coronavirus- 2 isolated in a tertiary care hospital. Psychiatry Res. 2020;289:113040. doi:10.1016/j. psychres.2020.113040 
25. Jeong H, Yim HW, Song Y-J, et al. Mental health status of people isolated due to Middle East respiratory syndrome. Epidemiol Health. 2016;38:e2016048-e2016048. doi:10.4178/epih.e2016048

26. Liu X, Kakade M, Fuller CJ, et al. Depression after exposure to stressful events: lessons learned from the severe acute respiratory syndrome epidemic. Compr Psychiatry. 2012;53(1):15-23. doi:10.1016/j.comppsych.2011.02.003

27. Yao H, Chen JH, Xu YF. Patients with mental health disorders in the COVID-19 epidemic. Lancet Psychiatry. 2020;7(4):e21. doi:10.1016/ S2215-0366(20)30090-0

28. Williams G, Di Nardo F, Verma A. The relationship between selfreported health status and signs of psychological distress within European urban contexts. Eur J Public Health. 2017;27 (suppl_2):68-73. doi:10.1093/eurpub/ckx008

29. Hamamura T, Mearns J. Depression and somatic symptoms in Japanese and American college students: negative mood regulation expectancies as a personality correlate. Int J Psychol. 2017;54 (3):351-359. doi:10.1002/ijop.12467

30. Lallukka T, Mekuria GB, Nummi T, Virtanen $P$, Virtanen $M$, Hammarström A. Co-occurrence of depressive, anxiety, and somatic symptoms: trajectories from adolescence to midlife using groupbased joint trajectory analysis. BMC Psychiatry. 2019;19(1):236. doi:10.1186/s12888-019-2203-7

31. Mostafaei S, Kabir K, Kazemnejad A, et al. Explanation of somatic symptoms by mental health and personality traits: application of Bayesian regularized quantile regression in a large population study. BMC Psychiatry. 2019;19(1):207. doi:10.1186/s12888-019-2189-1

32. Gupta MA. Review of somatic symptoms in post-traumatic stress disorder. Int Rev Psychiatry. 2013;25(1):86-99. doi:10.3109/ 09540261.2012.736367

33. Grupe DW, Nitschke JB. Uncertainty and anticipation in anxiety: an integrated neurobiological and psychological perspective. Nat Rev Neurosci. 2013;14(7):488-501.

34. Brooks SK, Webster RK, Smith LE, et al. The psychological impact of quarantine and how to reduce it: rapid review of the evidence. Lancet. 2020;395(10227):912-920. doi:10.1016/S0140-6736(20) 30460-8

35. Marjanovic Z, Greenglass ER, Coffey S. The relevance of psychosocial variables and working conditions in predicting nurses' coping strategies during the SARS crisis: an online questionnaire survey. Int $J$ Nurs Stud. 2007;44(6):991-998. doi:10.1016/j. ijnurstu.2006.02.012
36. Cava MA, Fay KE, Beanlands HJ, McCay EA, Wignall R. The experience of quarantine for individuals affected by SARS in Toronto. Public Health Nurs. 2005;22(5):398-406. doi:10.1111/ j.0737-1209.2005.220504.x

37. Bäuerle A, Graf J, Jansen C, et al. An e-mental health intervention to support burdened people in times of the COVID-19 pandemic: cope it. J Public Health (Oxf). 2020;fdaa058.

38. Webster RK, Brooks SK, Smith LE, Woodland L, Wessely S, Rubin GJ. How to improve adherence with quarantine: rapid review of the evidence. Public Health. 2020;182:163-169. doi:10.1016/j.puhe.20 20.03.007

39. Roma P, Monaro M, Muzi L, et al. How to improve compliance with protective health measures during the covid-19 outbreak: testing a moderated mediation model and machine learning algorithms. Int $J$ Environ Res Public Health. 2020;17(19):1-17. doi:10.3390/ijerph 17197252

40. Balanzá-Martínez V, Atienza-Carbonell B, Kapczinski F, De Boni RB. Lifestyle behaviours during the COVID-19 - time to connect. Acta Psychiatr Scand. 2020;141(5):399-400. doi:10.1111/acps.13 177

41. Mellick WH, Mills JA, Kroska EB, Calarge CA, Sharp C, Dindo LN. Experiential avoidance predicts persistence of major depressive disorder and generalized anxiety disorder in late adolescence. $J$ Clin Psychiatry. 2019;80(6):18m12265. doi:10.4088/JCP.18m12 265

42. Ottenbreit ND, Dobson KS, Quigley L. An examination of avoidance in major depression in comparison to social anxiety disorder. Behav Res Ther. 2014;56:82-90. doi:10.1016/j.brat.2014.03.005

43. Goethals L, Barth N, Guyot J, Hupin D, Celarier T, Bongue B. Impact of home quarantine on physical activity among older adults living at home during the COVID-19 pandemic: qualitative interview study (Preprint). JMIR Aging. 2020;3(1):e19007. doi:10.21 96/19007

44. Mattioli AV, Ballerini Puviani M, Nasi M, Farinetti A. COVID-19 pandemic: the effects of quarantine on cardiovascular risk. Eur J Clin Nutr. 2020;74(6):852-855. doi:10.1038/s41430-020-0646-Z

45. Kang C, Meng F, Feng Q, et al. Implementation of quarantine in China during the outbreak of COVID-19. Psychiatry Res. 2020;289 (113038):113038. doi:10.1016/j.psychres.2020.113038
Risk Management and Healthcare Policy

\section{Publish your work in this journal}

Risk Management and Healthcare Policy is an international, peerreviewed, open access journal focusing on all aspects of public health, policy, and preventative measures to promote good health and improve morbidity and mortality in the population. The journal welcomes submitted papers covering original research, basic science, clinical \& epidemiological studies, reviews and evaluations, guidelines, expert opinion and commentary, case reports and extended reports. The manuscript management system is completely online and includes a very quick and fair peer-review system, which is all easy to use. Visit http://www.dovepress.com/testimonials.php to read real quotes from published authors. 